\title{
A Characterization of Discretized Polygonal Convex Regions by Discrete Moments
}

\author{
Joviša Žunić‘ \\ Computer Science, Cardiff University, Queen's Buildings, \\ Newport Road, Cardiff CF24 3XF, Wales, U.K. \\ J.Zunic@cs.cf.ac.uk
}

\begin{abstract}
For a given planar region $P$ its discretization on a discrete planar point set $\mathcal{S}$ consists of the points from $\mathcal{S}$ which fall into $P$. If $P$ is bounded with a convex polygon having $n$ vertices and the number of points from $P \cap \mathcal{S}$ is finite, the obtained discretization of $P$ will be called discrete convex n-gon.

In this paper we show that discrete moments having the order up to $n$ characterize uniquely the corresponding discrete convex $n$-gon if the discretizing set $\mathcal{S}$ is fixed. In this way, as an example, the matching of discrete convex $n$-gons can be done by comparing $\frac{1}{2} \cdot(n+1) \cdot(n+2)$ discrete moments what can be much efficient than the comparison "point-by-point" since a digital convex $n$-gon can consist of an arbitrary large number of points.
\end{abstract}

Keywords: Discrete shape, coding, moments, pattern matching.

\section{Introduction}

It is known for many years that the moments are good descriptors of real shapes (48]). They are used in many computer vision, image processing, and pattern recognition tasks (25]). For simple "continuous" shapes as they are lines, circles, ellipses,... a finite set of moments is sufficient for recovering the original shape - usually, it is enough to solve a system of equations. If we try to reconstruct regions bounded by convex polygons (triangles, quadrangles,...) the problem of reconstruction of the original shape from a set of moments becomes more complicated but still solvable. The complication comes from the fact that there are no suitable equations for the boundaries of such polygonal convex regions.

But, in computer applications of the "moment techniques" we manipulate mostly with discrete data - not with real objects described by their equations. In areas as they are pattern recognition, pattern classification, (digital) image analysis, e.t.c., real objects are replaced with their discretizations - i.e., they are represented by finite point sets which are obtained by some discretizing process. It implies that there are infinitely many real shapes with the same discretization.

* The author is also with the Mathematical institute of Serbian Academy of Sciences and Arts, Belgrade. 


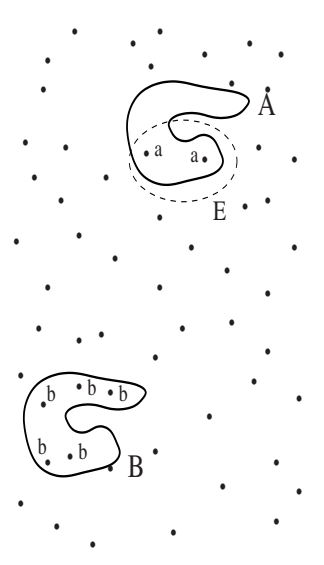

a) random grid

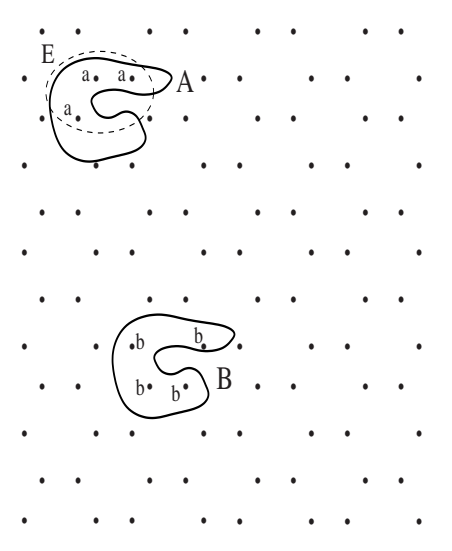

b) hexagonal grid

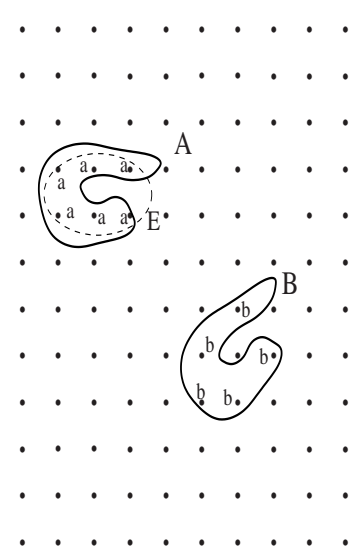

c) squared (integer) grid

Fig. 1. Discretizations of isometric shapes $A$ (points labeled by a) and $B$ (points labeled by b) on three different discretizing sets are shown. In all presented cases, the discretizations of $A$ and $B$ are non isometric, but discretizations of $A$ coincide with the discretizations of a given ellipse $E$.

So, even if we know an equation of the real object (what usually does not happen in the mentioned research areas), it is not suitable to represent the discrete image of a given real object by the corresponded equation because it can be happened that the considered digital object has many different characterizations (see discretizations of the shape $A$ from Fig. 1). More over, isometric planar regions may have non-isometric discretizations on the same discretizing set (see discretizations of the shapes $A$ and $B$ from Fig. 1) which also shows that the use of the "original objects" (sometimes called preimages) for a characterization of a given discrete object could be inappropriate.

Here, we prove that discrete moments having the order up to $n$ are enough for a unique characterization of discrete convex $n$-gons presented on a fixed discrete set. In this way, as an example, a fast comparison between discrete convex $n$-gons is enabled. In case of a relatively small number of edges of digitized convex polygons with respect to the number of sample points the comparison of the corresponded discrete moments can be much faster than than the comparison "point-by-point".

We conclude this introduction with the basic definitions and denotations.

By discrete convex $n$-gon we mean the discretization of a planar region which is bounded by a convex $n$-gon. Formally, a discrete convex $n$-gon $\mathbf{D}(P)$, (see Fig. 2) from a fixed discrete point set $\mathcal{S}$ is defined as

$$
\mathbf{D}(P)=\{(x, y) \mid(x, y) \in P \cap \mathcal{S}, \text { the boundary of region } P \text { is a convex } n \text {-gon }\} \text {. }
$$

Throughout the paper, it will be assumed but not mentioned, any appearing discrete convex $n$-gons consists of a finite number of points. For an illustration, 


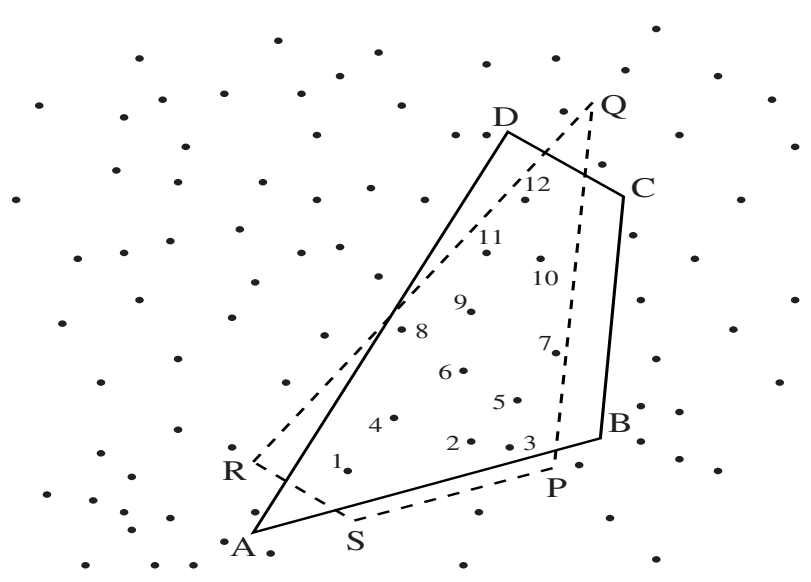

(a)

(b)

Fig. 2. (a) Discretizations of non isometric quadrangles $A B C D$ and $P Q R S$ are identical and they consist of 12 numbered points. (b) During the discretization process the vertices $A, B, C$, and $D$ (i.e., $P, Q, R$, and $S$ ) are usually unknown. We can only manipulate with the obtained discrete set (i.e., discrete 4 -gon).

the discretizations on the set consisting of all points with the coordinates which are rational numbers (i.e., $\mathcal{S}=\mathbf{Q}^{2}$ ) are not considered.

Since the characterization of discrete convex $n$-gons described here is based on a suitable use of discrete moments we give a precise definition. The discrete moment $\mu_{p, q}(X)$ of a finite number point set $X$ is:

$$
\mu_{p, q}(X)=\sum_{(x, y) \in X} x^{p} \cdot y^{q} .
$$

The moment $\mu_{p, q}(X)$ has the order $p+q$. In the rest of the paper it will be assumed (even not mentioned) that $p$ and $q$ are nonnegative integers. The set of nonnegative integers is denoted by $\mathbf{N}_{\mathbf{o}}$.

Through the paper a finite set means a set consisting of a finite number of points. Also, a unique characterization and a coding will have the same meaning.

We shall say that a continuous function $z=f(x, y)$ separates sets $A$ and $B$ if the sign of $f(x, y)$ in the points from $A$ differs from the sign of $f(x, y)$ in the points from $B$. Precisely, it is

$\begin{array}{llll}\text { either } & A \subset\{(x, y) \mid f(x, y)>0\} & \text { and } & B \subset\{(x, y) \mid f(x, y)<0\}, \\ \text { or } & A \subset\{(x, y) \mid f(x, y)<0\} & \text { and } & B \subset\{(x, y) \mid f(x, y)>0\} .\end{array}$

Some examples are given on Fig. 3 .

\section{Characterization of Discrete Convex $\boldsymbol{n}$-gons}

In this section it will be shown that the discrete moments having order up to $n$ match uniquely the discretized polygonal convex $n$-gon presented on a fixed set $\mathcal{S}$. We start with the following theorem. 


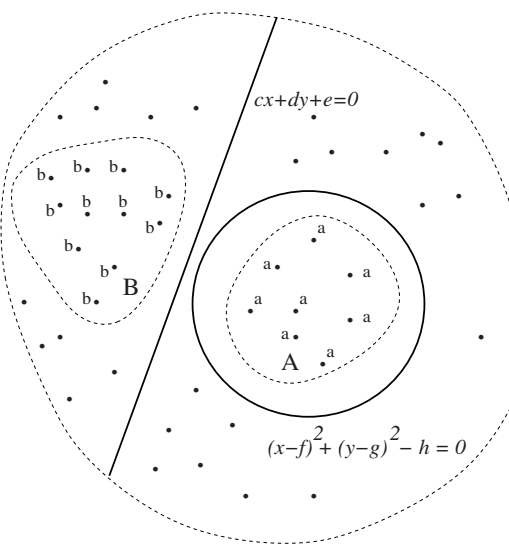

(a)

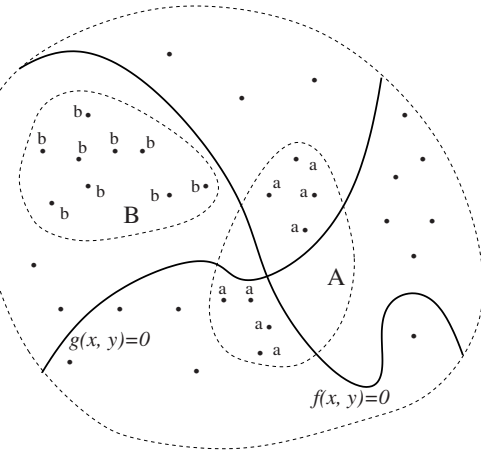

(b)

Fig. 3. (a) $z=c \cdot x+d \cdot y+e$ separates discrete sets $A$ (points labeled by $a$ ) and $B$ (points labeled by $b) . z=(x-f)^{2}+(y-g)^{2}-h$ also separates $A$ and $B$. But, $z=(c \cdot x+d \cdot y+e) \cdot\left((x-f)^{2}+(y-g)^{2}-h\right)$ does not separate $A$ and $B$.

(b) $z=f(x, y)$ does not separate the sets $A$ (points labeled by $a$ ) and $B$ (points labeled by $b)$. $z=g(x, y)$ also does not separate $A$ and $B$. But, $z=f(x, y) \cdot g(x, y)$ separates $A$ and $B$.

Theorem 1. Let $A_{1}$ and $A_{2}$ be two finite planar sets. If there exists a function of the form

$$
f(x, y)=\sum_{p+q \leq k} \alpha_{p, q} \cdot x^{p} \cdot y^{q}, \quad \text { where } \quad p, q \in \mathbf{N}_{\mathbf{o}}
$$

which separates $A_{1} \backslash A_{2}$ and $A_{2} \backslash A_{1}$ then

$$
\mu_{p, q}\left(A_{1}\right)=\mu_{p, q}\left(A_{2}\right) \quad \text { with } \quad p, q \in \mathbf{N}_{\mathbf{o}} \quad \text { and } \quad p+q \leq k
$$

is equivalent to

$$
A_{1}=A_{2} \text {. }
$$

Proof. If $A_{1}=A_{2}$ then the corresponding discrete moments are equal obviously. It remains to prove that the equality of the corresponded moments of the order up to $k$ preserves $A_{1}=A_{2}$. We prove that $A_{1} \neq A_{2}$ and

$$
\sum_{(x, y) \in A_{1}} x^{p} \cdot y^{q}=\mu_{p, q}\left(A_{1}\right)=\mu_{p, q}\left(A_{2}\right)=\sum_{(x, y) \in A_{2}} x^{p} \cdot y^{q}
$$

(satisfied for all $p, q \in \mathbf{N}_{\mathbf{o}}$ with $p+q \leq k$ ) lead to a contradiction. Since $A_{1} \neq A_{2}$ we can assume $A_{1} \backslash A_{2} \neq \emptyset$ (else we can start with $A_{2} \backslash A_{1} \neq \emptyset$ ). Further, because there exists a function $f(x, y)=\sum_{p+q \leq k} \alpha_{p, q} \cdot x^{p} \cdot y^{q}$ which separates $A_{1} \backslash A_{2}$ and $A_{2} \backslash A_{1}$, we can assume (for instance) $f(x, y)>0$ if $(x, y) \in A_{1} \backslash A_{2}$, while $(x, y) \in A_{2} \backslash A_{1}$ implies $f(x, y)<0$. Now, we are able to derive the contradiction $0<0$ which finishes the proof.

$$
0<\sum_{(x, y) \in A_{1} \backslash A_{2}} f(x, y)-\sum_{(x, y) \in A_{2} \backslash A_{1}} f(x, y)
$$




$$
\begin{aligned}
& =\sum_{p+q \leq k}\left(\sum_{(x, y) \in A_{1} \backslash A_{2}} \alpha_{p, q} \cdot x^{p} \cdot y^{q}+\sum_{(x, y) \in A_{1} \cap A_{2}} \alpha_{p, q} \cdot x^{p} \cdot y^{q}\right) \\
& -\sum_{p+q \leq k}\left(\sum_{(x, y) \in A_{2} \backslash A_{1}} \alpha_{p, q} \cdot x^{p} \cdot y^{q}+\sum_{(x, y) \in A_{1} \cap A_{2}} \alpha_{p, q} \cdot x^{p} \cdot y^{q}\right) \\
& =\sum_{p+q \leq k}\left(\alpha_{p, q} \cdot \sum_{(x, y) \in A_{1}} x^{p} \cdot y^{q}-\alpha_{p, q} \cdot \sum_{(x, y) \in A_{2}} x^{p} \cdot y^{q}\right)=0 .
\end{aligned}
$$

Next, we prove the main result of the paper.

Theorem 2. Fix a discrete point set $\mathcal{S}$. Let given discrete convex $n$-gons $\mathbf{D}(P), \mathbf{D}\left(P_{1}\right) \subset \mathcal{S}$. Then

$$
\left(\mu_{p, q}(\mathbf{D}(P))=\mu_{p, q}\left(\mathbf{D}\left(P_{1}\right)\right) \text { with } p+q \leq n, p, q \in \mathbf{N}_{\mathbf{o}}\right) \quad \Longrightarrow \quad \mathbf{D}(P)=\mathbf{D}\left(P_{1}\right) \text {. }
$$

Proof. Let $\mathbf{D}(P)$ and $\mathbf{D}\left(P_{1}\right)$ be different discrete convex $n$-gons. We will show that there always exists a separating function of the form (1) (with $k \leq n$ ) which separates $\mathbf{D}(P) \backslash \mathbf{D}\left(P_{1}\right)$ and $\mathbf{D}\left(P_{1}\right) \backslash \mathbf{D}(P)$. Then, the statement will follow directly form Theorem 1 , specifying $A_{1}=\mathbf{D}(P)$ and $A_{2}=\mathbf{D}\left(P_{1}\right)$.

Since $\mathbf{D}(P) \neq \mathbf{D}\left(P_{1}\right)$ and $\mu_{0,0}(\mathbf{D}(P))=\mu_{0,0}\left(\mathbf{D}\left(P_{1}\right)\right)$ are assumed, we have $\mathbf{D}(P) \backslash \mathbf{D}\left(P_{1}\right) \neq \emptyset$ and $\mathbf{D}\left(P_{1}\right) \backslash \mathbf{D}(P) \neq \emptyset$.

For convenience and without loss of generality we can assume that $P$ and $P_{1}$ do not have common vertices and common edges, but also that there is no edge of $P$ (i.e., $P_{1}$ ) which belongs to the boundary of $P_{1}$ (i.e., $P$ ) - such an assumption is possible because $\mathbf{D}(P)$ and $\mathbf{D}\left(P_{1}\right)$ are finite number point sets.

Let we consider the set-intersection of $P$ and $P_{1}$ and let

$$
\begin{gathered}
A_{1}, A_{2}, \ldots, A_{i_{1}} \equiv B_{1}, B_{2}, \ldots, B_{j_{1}} \equiv \\
\equiv A_{i_{1}+1}, A_{i_{1}+2}, \ldots, A_{i_{2}} \equiv B_{j_{1}+1}, B_{j_{1}+2}, \ldots, B_{j_{2}} \\
\ldots \\
\ldots \\
A_{i_{k-1}+1}, A_{i_{k-1}+2}, \ldots, A_{i_{k}} \equiv B_{j_{k-1}+1}, B_{j_{k-1}+2}, \ldots, B_{j_{k}} \equiv A_{1}
\end{gathered}
$$

be the vertices of $P \cap P_{1}$ listed in the counterclockwise order and denoted in such a way that the line segments

$$
\begin{array}{r}
{\left[A_{1}, A_{2}\right], \ldots,\left[A_{i_{1}-1}, A_{i_{1}}\right],} \\
{\left[A_{i_{1}+1}, A_{i_{1}+2}\right], \ldots,\left[A_{i_{2}-1}, A_{i_{2}}\right],} \\
\ldots \ldots \ldots, \\
{\left[A_{i_{k-1}+1}, A_{i_{k-1}+2}\right], \ldots,\left[A_{i_{k}-1}, A_{i_{k}}\right]}
\end{array}
$$

belong to the boundary of $P$, while

$$
\begin{array}{r}
{\left[B_{1}, B_{2}\right], \ldots,\left[B_{j_{1}-1}, B_{j_{1}}\right],} \\
{\left[B_{j_{1}+1}, B_{j_{1}+2}\right], \ldots,\left[B_{j_{2}-1}, B_{j_{2}}\right],} \\
\ldots \ldots \ldots, \\
{\left[B_{j_{k-1}+1}, B_{j_{k-1}+2}\right] \ldots,\left[B_{j_{k}-1}, B_{j_{k}}\right]}
\end{array}
$$


belong to the boundary of $P_{1}$. Further, let

- $\alpha_{1} \cdot x+\beta_{1} \cdot y-\gamma_{1}=0$ be the line $l_{1}$ determined by $A_{1}$ and $A_{i_{1}}$;

$-\alpha_{2} \cdot x+\beta_{2} \cdot y-\gamma_{2}=0$ be the line $l_{2}$ determined by $A_{i_{1}+1}$ and $A_{i_{2}}$;

$-\alpha_{k} \cdot x+\beta_{k} \cdot y-\gamma_{k}=0$ be the line $l_{k}$ determined by $A_{i_{k-1}+1}$ and $A_{i_{k}}$. Then we will show that the function

$$
f(x, y)=\prod_{i=1}^{k}\left(\alpha_{i} \cdot x+\beta_{i} \cdot y-\gamma_{i}\right)
$$

separates the set-differences $\mathbf{D}(P) \backslash \mathbf{D}\left(P_{1}\right)$ and $\mathbf{D}\left(P_{1}\right) \backslash \mathbf{D}(P)$.

Namely, since the points

$$
A_{1}, A_{i_{1}}, A_{i_{1}+1}, A_{i_{2}}, A_{i_{k-1}+1}, A_{i_{k}}
$$

are successive intersection points of the boundaries of $P$ and $P_{1}$, we have:

i) for any $i$ from $\{1,2, \ldots, k\}$, all points from $\mathbf{D}(P) \backslash \mathbf{D}\left(P_{1}\right)$ belong to the same half-plane determined by the line $l_{i}$ - consequently, $f(x, y)$ in all points from $\mathbf{D}\left(P_{1}\right) \backslash \mathbf{D}(P)$ takes the same sign;

ii) for any point $X$ from $\mathbf{D}\left(P_{1}\right) \backslash \mathbf{D}(P)$ there is exactly one integer $i$ from $\{1, \ldots, n\}$ such that $l_{i}$ separates $X$ from $\mathbf{D}(P) \backslash \mathbf{D}\left(P_{1}\right)$. In other words, the function $f(x, y)$ takes the same sign in all points from $\mathbf{D}\left(P_{1}\right) \backslash \mathbf{D}(P)$ and the sign differs from the sign taken in the points from $\mathbf{D}(P) \backslash \mathbf{D}\left(P_{1}\right)$.

The items i) and ii) imply that $f(x, y)$ is a separating function for $\mathbf{D}(P) \backslash \mathbf{D}\left(P_{1}\right)$ and $\mathbf{D}\left(P_{1}\right) \backslash \mathbf{D}(P)$.

Let us mention here that another separating function for the same setdifferences is

$$
\tilde{f}(x, y)=\prod_{i=1}^{k}\left(\tilde{\alpha}_{i} \cdot x+\tilde{\beta}_{i} \cdot y-\tilde{\gamma}_{i}\right)
$$

where

- $\tilde{\alpha}_{1} \cdot x+\tilde{\beta}_{1} \cdot y-\tilde{\gamma}_{1}=0$ is the line $\tilde{l}_{1}$ determined by $B_{1}$ and $B_{j_{1}}$;

$-\tilde{\alpha}_{2} \cdot x+\tilde{\beta}_{2} \cdot y-\tilde{\gamma}_{2}=0$ is the line $\tilde{l}_{2}$ determined by $B_{j_{1}+1}$ and $B_{j_{2}}$;

$-\tilde{\alpha}_{k} \cdot x+\tilde{\beta}_{k} \cdot y-\tilde{\gamma}_{k}=0$ is the line $\tilde{l}_{k}$ determined by $B_{j_{k-1}+1}$ and $B_{j_{k}}$.

Due to Theorem 1, the existence of a function of the form (1) which separates $\mathbf{D}(P) \backslash \mathbf{D}\left(P_{1}\right)$ and $\mathbf{D}\left(P_{1}\right) \backslash \mathbf{D}(P)$ (we can take either $(2)$ or $(3)$ ) completes the proof. [|

The previous proof is illustrated by Fig. 4 . In the given example $n=7, k=3$, and two separating functions are described in the capture of the figure. 


\section{Comments and Conclusion}

In this paper we consider finite number point subsets of a fixed discrete point set $\mathcal{S}$ which are occupied by planar regions whose boundaries are convex $n$-gons. Such sets are called discrete convex $n$-gons. Through the manuscript, there is no any assumption about the structure of $\mathcal{S}$. We derive a result which shows that there are no two different discrete convex $n$-gons whose corresponded discrete moments of the order up to $n$ coincide. Or, more formally, the mapping

$$
\begin{aligned}
\mathbf{D}(P) \longrightarrow & \left(\begin{array}{l}
\mu_{0,0}(\mathbf{D}(P)), \mu_{0,1}(\mathbf{D}(P)), \mu_{1,0}(\mathbf{D}(P)), \ldots, \\
\left.\mu_{0, n}(\mathbf{D}(P)), \mu_{1, n-1}(\mathbf{D}(P)), \ldots, \mu_{n, 0}(\mathbf{D}(P))\right)
\end{array}\right.
\end{aligned}
$$

is one-to-one while $\mathbf{D}(P)$ belongs to the set of digital convex n-gons.

A precise performance analysis could not be given since there are no assumption about the structure of $\mathcal{S}$. In any case, it can be said that the result enables the matching of discrete convex $n$-gons by comparing $\frac{(n+1) \cdot(n+2)}{2}$ numbers (which are all discrete moments of the order up to $n$ ) instead of the comparing "pointby-point". Obviously, the comparison "point-by-point" can be very expensive

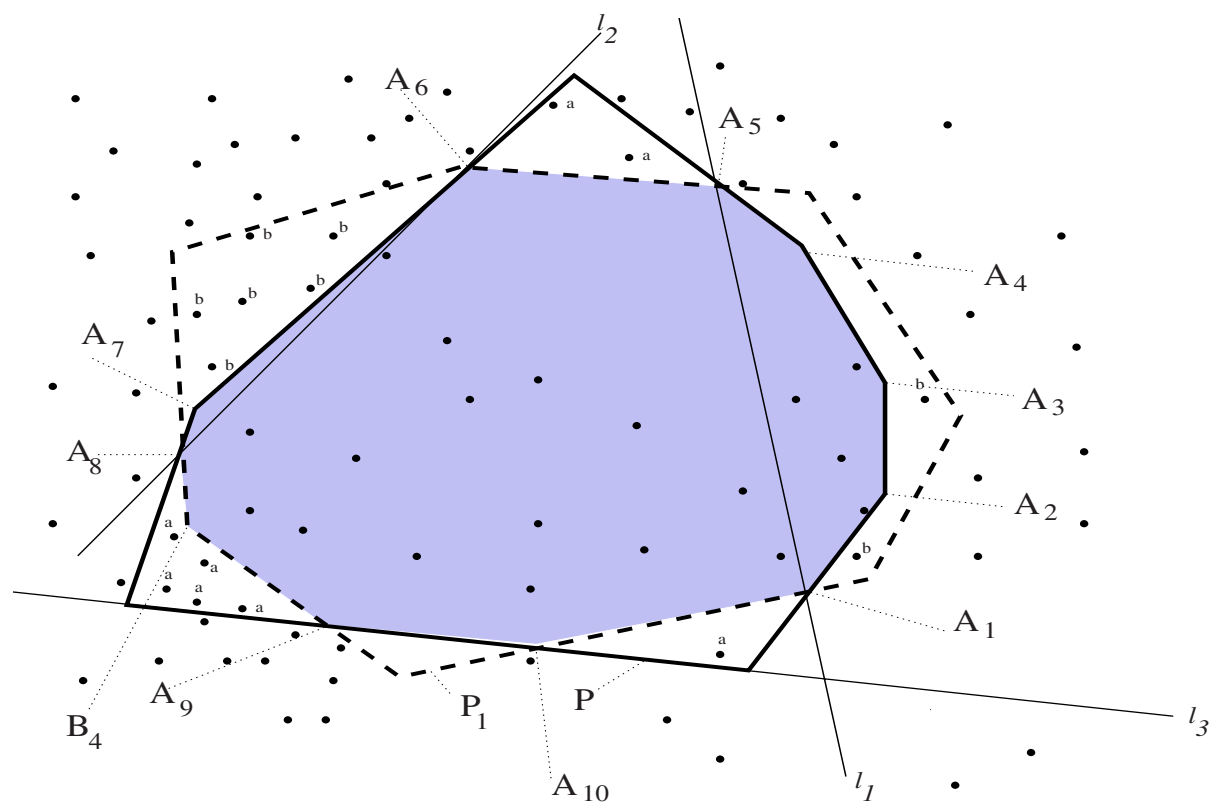

Fig. 4. $f(x, y)=\prod_{i=1}^{i=3}\left(\alpha_{l_{i}} \cdot x+\beta_{l_{i}} \cdot y-\gamma_{l_{i}}\right)$ is a separating function for $\mathbf{D}(P) \backslash \mathbf{D}\left(P_{1}\right)$ (points labeled by $a$ ), and $\mathbf{D}\left(P_{1}\right) \backslash \mathbf{D}(P)$ (points labeled by $b$ ).

In accordance with denotations from the proof of Theorem $2, B_{1} \equiv A_{5}, B_{2} \equiv A_{6}$, $B_{3} \equiv A_{8}, B_{5} \equiv A_{9}, B_{6} \equiv A_{10}$, and $B_{7} \equiv A_{1}$. The function which is the product of the linear functions corresponded to lines determined by pairs of points $\left(B_{1}, B_{2}\right),\left(B_{3}, B_{5}\right)$, and $\left(B_{6}, B_{7}\right)$ is also a function which separates $\mathbf{D}(P) \backslash \mathbf{D}\left(P_{1}\right)$ and $\mathbf{D}\left(P_{1}\right) \backslash \mathbf{D}(P)$. 
because the discrete convex $n$-gons may consist of an arbitrary large number of points. In areas of practical applications $\mathcal{S}$ can correspond to the set of sensors or to the set of reference points whose choice depends of the nature of application.

Of course, more precise analysis can be done in the area of digital image analysis - i.e., if $\mathcal{S}$ is an integer (squared) grid (it is planned as a future work). In the literature, the problems related to digital squares are already considered. In 39 the recognition problem is studied.

It is worth to mention that the representation (coding) problem for digital convex polygons from an integer grid can be solved by decomposing the boundary of the considered digital polygon onto digital straight line segments and, after that, to characterize (code) the obtained straight line segments. Both, efficient algorithms for the decomposition of digital curves into maximal straight line segments and efficient coding scheme for digital straight line segments already exist - see 610 and [17. Let us notice, that the coding scheme presented here is expected to be more robust because it is not based on the boundary points only, as it would be in the case of the coding based on the boundary decomposition into digital straight line segments. Also, such a coding procedure could not be applied to discrete convex polygons from an arbitrary discretizing set.

\section{References}

1. Dorst, L., Smeulders, A. W. M.: Discrete representation of straight lines. IEEE Trans. Pattern Anal. Machine Intell. 6 (1984) 450-463.

2. Duda, R. O., Hart, P. E.: Pattern Classification and Scene Analysis. Wiley, New York, USA, 1973.

3. Forchhammer, S., Kim, C. E.: Digital squares. IEEE Trans. Pattern Anal. Machine Intell. 22 (1988) 672-674.

4. Hu, M.: Visual pattern recognition by moment invariants, IRE Trans. Inf. Theory 8 (1962) 179-187.

5. Jain, R., Kasturi, R., Schunck, B. G.: Machine Vision. McGraw-Hill, New York, USA, 1995.

6. Lindenbaum, M., Bruckstein, A.: "On recursive, $\mathcal{O}(N)$ partitioning of a digitized curve into digital straight segments," IEEE Trans. Pattern Anal. Machine Intell. 15 (1993) 949-953.

7. Lindenbaum, M., Koplowitz, J.: A new parametrization of digital straight lines. IEEE Trans. Pattern Anal. Machine Intell. 13 (1991) 847-852.

8. Mamistvalov, A. G.: $n$-Dimensional moment invariants and conceptual mathematical theory of recognition $n$-dimensional solids. IEEE Trans. Pattern Anal. Machine Intell. 20 (1998) 819-831.

9. Nakamura, A., Aizawa, K.: Digital squares. Computer Vision, Graphics Image Processing 49 (1990) 357-368.

10. Smeulders, A. W. M., Dorst, L.: "Decomposition of discrete curves into piecewise straight segments in linear time," in Vision Geometry (P. Bhattacharya, A. Rosenfeld, eds.), American Mathematical Society, series Contemporary Mathematics, 119 (1991) 169-195. 